\title{
INVESTIGATION AND EVALUATION OF IEEE 802.11N WLANs LiNK FEATURES PERFORMANCE UNDER SINGLE HOST AND CONCURRENT COMMUNICATION
}

\author{
Sumon Kumar Debnath ${ }^{1}$ Prodip Kumar Sarker ${ }^{2}$, Md. Manowarul Islam ${ }^{3}$, and \\ Ileas Pramanik ${ }^{2}$ \\ ${ }^{1}$ Department of Electrical and Electronic Eng., Begum Rokeya University, Bangladesh \\ ${ }^{2}$ Department of Computer Science and Eng., Begum Rokeya University, Bangladesh \\ ${ }^{3}$ Department of Computer Science and Eng., Jagannath University, Bangladesh
}

\begin{abstract}
For an efficient design of wireless local-area networks (WLANs), the simulation tools are important to accurately estimate the IEEE 802.11n/ac link features for WLANs. However, this true simulation of network behavior is critical in designing high-performance WLANs. Through testing, analysis, and modeling of the proposed scheme repetitively, the design of the WLAN can be enhanced with a small budget before making its practical implementation. Many network simulation tools have been established to give solutions for this request and $n s-3$ is the most widely used tools among them by the research industry as an open-source network simulator. In this paper, we examine the various link features of the 802.11n WLANs under several conditions. We investigate the effects of 802.11n WLAN modulation and coding schemes (MCSS), 20MHz single channel or $40 \mathrm{MHz}$ bonded channel, guard intervals (GI), frame aggregation, data encoding, number of antennas and their data rate, and link distance features of 802.11n WLAN in ns-3 when only a unique host connects with the access point (AP) and generates data traffic. Besides, the performance for an enterprise scenario proposed by the IEEE 802.11ax study group is evaluated when several hosts are simultaneously creating traffic with their associated APs. The results demonstrate that ns-3 support most of the link features of the 802.11n protocol with significant accuracy.
\end{abstract}

\section{KEYWORDS}

IEEE 802.11n, MIMO, SISO, neighboring access-point association, Algorithm, ns-3 simulator.

\section{INTRODUCTION}

Currently, the IEEE $802.11 \mathrm{n} / \mathrm{ac}$ WiFi standard has commonly installed on most customer devices like a variety of laptop PCs, smartphones, tablets, and access points (APs) due to their small price, flexible and high-performance Internet accessed services [1] [2]. Among them, the IEEE $802.11 \mathrm{n}$ is the most commonly used protocol in wireless local-area networks (WLANs). It approves several new technologies such as the number of antennas employed in Multiple Input Multiple Output (MIMO) devices, the $40 \mathrm{MHz}$ bonded channel, the aggregation of the frame, and the block acknowledgment mechanism that can increase the data transmission capacity up to $600 \mathrm{Mbps}$ [3] [4]. Thus, the IEEE $802.11 \mathrm{n}$ protocol should be focused on.

In WLAN, a host normally uses a laptop/desktop PC, or a mobile device that can generate traffic by single-host communication or multiple-hosts concurrent communication with the AP at a time. In the scenario of concurrent traffic, multiple hosts send data simultaneously with one AP. During concurrent communication, the rate of packet collision increases with the amount of 
communicating hosts per AP and that makes throughput drops significantly [5]. In this case, a host which is adjacent to the AP might have higher data transmission possibility than the remote hosts.

In many cases, the WLAN researchers and engineers should need to test the link features of 802.11n protocols, hardware, and scenarios through simulation studies before implementing them in a practical environment. This accurate simulation of link features of IEEE $802.11 \mathrm{n}$ is a very critical matter in developing an innovative model or algorithms for WLAN [6] [7] [8], mobile ad-hoc network (MANET) [9] [10] or vehicular ad-hoc networks (VANET) [11]. Using repetitive modeling, and simulation of any proposed algorithm through network simulator, the IEEE $802.11 \mathrm{n}$ WLANs system design can be enhanced cost-effectively before its physical deployment in real environments. Several network simulators have been proposed to give their solutions. They include WIMNET simulator [12], ns-2 [13], ns-3 [14], QualNet [15], and OPNET [16]. Among them, ns-3 has been widely used in academic societies to evaluate new protocols and techniques for WLANs as a realistic non-proprietary software platform. Therefore, it is significant to study the various link features of the $802.11 \mathrm{n}$ protocol through ns3 simulations and evaluate their performance accuracy under different operating conditions.

In this paper, we evaluate the performance of various link features of 802.11n WLANs using ns3 simulation. We investigate different key features such as the throughput and maximum feasible range with modulation coding schemes (MCSs), frame aggregation, long/short GI, channel bonding, link distance performance, and the impact of the number of antennas in Single Input Single Output (SISO) or MIMO links and their data rate through ns-3 simulation when considering only one host is sending to or receiving packets from one AP. We also evaluate the effect of concurrent communication by multiple hosts per AP in an enterprise network scenario.

We organize the rest of the paper as follows: Section 2 inspects some related works relevant to our research. Section 3 reviews the 802.11n WLANs protocol and its link features. Section 4 briefly mentions the procedure of the neighboring AP association approach used for finding hosts association for an enterprise network. Section 5 shows an overview of the simulation environment for performance evaluation. Section 6 and section 7 explores the performance evaluation results by simulation. Lastly, Section 8 concludes the paper with some future recommendations.

\section{RELATED WORKS}

Currently, numerous research activities are carried out in literature to examine various performance issues of $802.11 \mathrm{n} / \mathrm{ac}$ protocol for WLANs through simulation. In this section, we concisely review some related previous works relevant to our paper.

In [17], the authors evaluate the comparative performance features of the 802.11ac WLAN relative to $802.11 \mathrm{n}$ protocol using ns-3.24.1 by considering several links features like channel bonding $(20 / 40 \mathrm{MHz}$ channel in $802.11 \mathrm{n}$ and $20 / 40 / 80 / 160 \mathrm{MHz}$ channel in $802.11 \mathrm{ac}$ ), guard intervals (long/short) and MCSs (MCS-0 MCS-7/MCS-9) while evaluating HT/VHT throughput, jitters, and delay. In the simulation, they did not evaluate all link features of the protocol such as SU-MIMO and MU-MIMO link features, the influence of frame aggregation, rate adaptation WiFi manager, and channel interference effects. Besides, they assume only a single client/host communication scenario with the Access Point (AP) through ns-3 simulation. But in a real network environment, concurrent traffic generation by multiple hosts with an AP may have different performance features which should be analyzed through ns-3 simulation.

In [18], the throughput estimation model for the 802.11n MIMO link in WLANs is investigated by considering a single-host and multiple-hosts communications scenario with an AP. They 
verified their model accuracy by comparing the measured throughput results with the estimated one using their throughput estimation model through WIMNET simulation [8].

In [19], the effect of Quality of Service (QoS) on the performance enhancement of the 802.11n $4 \times 4$ MIMO WLAN with random network topology for multimedia traffic is investigated using OPNET modular. In [20], authors evaluate the efficacy of 802.11n standard over the longdistances network in terms of 802.11a link using ns-3 simulation. Through simulation, they validate the efficiency of $802.11 \mathrm{n}$ PHY and MAC layer performance by evaluating several simulation scenarios like frame aggregation, data rates, bandwidth, and distances.

In [21], authors made modifications to the existing PHY model of the ns-3 simulator to support for wider channels together with the bit-error-rate (BER) estimations with higher MCSs for 802.11 ac WLAN. They evaluated these modifications using various simulation scenarios for the traffic generated by a single client with an AP. They also examined the performance of $802.11 \mathrm{ac}$ for the multiple client concurrent communication situations carried out in an enterprise network scenario from the upcoming IEEE 802.11ax work-group where multiple clients produced traffic simultaneously to their associate APs. However, in this study, the SU-MIMO and MU-MIMO and beam-forming link feature for $802.11 \mathrm{ac} / \mathrm{n}$ standard were not implemented and evaluated through simulation.

\section{OVERVIEW OF IEEE 802.11N STANDARD}

The 802.11n protocol mainly builds on the former 802.11 standards which include various novel technologies into it. They include multiple-antennas MIMO concepts at the access point (AP) and at the client PC side of the network, $40 \mathrm{MHz}$ channel bonding by combining two adjacent $20 \mathrm{MHz}$ channels and aggregated multiple frames into one frame before packet transmission to enhance its communication performance [2]. In this section, we briefly review the link features of the $802.11 \mathrm{n}$ protocol.

In 802.11n WLAN, the MIMO technology uses multiple antennas to concurrently transmit and/or receive more information up to four times than the SISO link. It can afford this by using Spatial Division Multiplexing (SDM), which can enhance the throughput significantly by raising the number of spatial streams (SSs) [3]. Because each SS involves a separate transmitting and receiving antenna. The $802.11 \mathrm{n}$ standards support $1 \times 1$ to $4 \times 4$ antennas. For example, the $802.11 \mathrm{n}$ can provide the maximum $600 \mathrm{Mbps}$ throughput with the bonded channel of $40 \mathrm{MHz}$, the short guard interval (GI) of 400ns, and with the $4 \times 4$ MIMO technology [3].

The $802.11 \mathrm{n}$ link can be operated in both $5 \mathrm{GHz}$ or $2.4 \mathrm{GHz}$ RF band and compatible with the former standard $(802.11 \mathrm{a} / \mathrm{b} / \mathrm{g})$. Besides working with $20 \mathrm{MHz}$ channels, it can support channel bonding (CB) to double the physical-layer (PHY) data rate where two adjacent $20 \mathrm{MHz}$ channels are worked together to make one broader channel of $40 \mathrm{MHz}$. The MIMO architecture together with the CB can further increase the physical data rate [22]. However, the CB concept has a higher potential for interference with other systems using the same frequencies in 2.4 GHz.

In the $802.11 \mathrm{n}$ standard, various modulations, error-correcting codes, and the number of SSs used, denoted by MCSs index, which is called mode. The 802.11n standard outlines 31 modes (MCS-0 MCS-31). Through using orthogonal frequency division multiplexing (OFDM), this protocol offers more immunity counter to the selective fading. In $802.11 \mathrm{n}$, the amount of OFDM sub-carriers is also improved. For example, with the $20 \mathrm{MHz}$ channel the total OFDM sub-carriers are 56 (52 usable) to support High Throughput (HT) and with the $40 \mathrm{MHz}$ channel bonding it can be improved up to 114 (108 usable) in HT. Every sub-carrier is modulated using a suitable modulation type (BPSK, QPSK, 16-QAM, or 64-QAM) and forward error correction 
coding rate of $1 / 2,2 / 3,3 / 4$, or $5 / 6$ [3]. The GI is reduced by half (400ns) between OFDM symbols to afford more data throughput.

The 802.11n protocol supports frame aggregation and blocked acknowledgment (ACK) mechanism to provide its MAC layer enhancements. With frame aggregation, we can increase the data rate at the user level by aggregating multiple data frames into one for transmission at each occurrence to cut the overhead of the protocol. The 802.11n protocol supports two types of aggregation namely 1) MAC protocol data unit (A-MPDU) and 2) the MAC service data unit (A-MSDU) [3].

\section{Neighboring Access Point (NAP) Association Algorithm for EVALUATION OF AN ENTERPRISE NETWORK}

In this section, we describe the procedure of the neighboring AP association algorithm to find the set of associated hosts to each AP for an enterprise network.

1. We calculate the throughput between each of the available candidates APs and hosts through the throughput estimation model in [18] as:
A. First, we compute the Euclid distance for each possible pair of APs and a host.
B. Then, we estimate received signal strength (RSS) between them via the log distance model.
C. Finally, we convert the RSS into the throughput.

2. Drive the associable AP for a host with the largest link speed by the greedy approach as:

A. For each host, select a candidate AP such that the RSS at the host is maximum.

B. Associate the host to the AP in step 2. (A) with the largest link speed.

3. Repeat step 2 until each host is associated with an AP.

\section{Simulation Environment For Performance Evaluation}

Network simulator ns-3 is used to evaluate various link features of the IEEE 802.11n WLANs MIMO/SISO protocol. ns-3 is the discrete-event network simulator commonly used in academic societies as an openly accessible software tool to model any new protocols, techniques, and algorithms for WLANs [14]. In this paper, we use the ns-3.28 version that was released in March 2018. The code has been developed by $\mathrm{C}++$, and the scenario for network simulations can be written by Python. It has been developed as the simulation engine by many users in the study and research of wired and wireless LANs. Currently, ns-3 adopts the key feature of IEEE $802.11 \mathrm{n} / \mathrm{ac}$ or others protocol such as Multiple-Input-Multiple-Output (MIMO), the channel bonding, long and short GI, and the MSDU or MPDU frame aggregation that can be useful to model and evaluate various algorithms for WLANs in 2.4 and $5 \mathrm{GHz}$. For performance evaluation, we consider two network scenarios by simulation: 1) Traffic created between a unique host and the AP to form WLAN and 2) traffic produced concurrently by many hosts with their associated APs. In the first scenario, a single host is associated and generated traffic with the AP, forming 802.11n WLAN. In this scenario, we investigate the effect of data throughput and transmission range with different MCSs (802.11n allows MCS-0 to MCS-31), link distance features by rate adaptation, doubling the bandwidth of $802.11 \mathrm{n}$ channels $(802.11 \mathrm{n}$ agrees 20 $\mathrm{MHz}$ channel or $40 \mathrm{MHz}$ bonded channel), the number of antennas proportional to the simultaneous data streams at both sides of the link (802.11n permits $1 \times 1$ to $4 \times 4$ antennas), the long or short GI, and the maximum size of the aggregated frame.

In the second scenario, we consider an enterprise network scenario where multiple hosts are associated with an AP and concurrently generating traffic with it. Here, we examine the average throughput performance by increasing the numbers of active hosts that are concurrently 
communicating with their associated APs. In both scenarios, we estimate the throughput for every HT bit rate value as the main performance metric which is calculated using the flow monitor [22] as

$$
\text { Throught }=\frac{\text { rxBytes } * 8}{\text { TimeLastRxpkt }- \text { TimeFirstR xpkt }}
$$

Where rxBytes is the total number of received packets, TimeLastRxPacket is the absolute time of the last received packet and TimeFirstRxPacket does the absolute time of the first received packet by flow monitor.

\section{Evaluation Results for Traffic Produced between A Unique HOST AND AN AP}

In this portion, we show various simulation results when a unique host makes traffic with AP.

\subsection{Average throughput and maximum range with various MCSs}

In the first simulation, we investigate the relationship between peak throughput and transmission range with different MCSs and various physical distances between the AP and a host. Table 1 shows the simulation parameters and Figure 1 demonstrates the simulation results. From Figure 1 it is observed that although the peak throughput rises with increasing the MCSs value, it decreases its communication range. This lower range with higher MCSs index values is expected because the higher MCSs require more signal-to-noise ratio (SNR) value so that the receiver can successfully understand them and signals pass through longer distances tend to become weak that offer inferior SNRs.

Table 1. Parameters for transmission range simulation.

\begin{tabular}{|l|l|}
\hline Parameter & Value \\
\hline Propagation Model & constant position model \\
\hline mobility model & log distance model \\
\hline remote station manager & constant rate manager \\
\hline packet size & UDP, 1472 bytes \\
\hline error rate model & YansErrorRateModel \\
\hline$\#$ of spatial streams & $1 \times 1,2 \times 2$ \\
\hline Tx power & $16.0206 \mathrm{dBm}$ \\
\hline Mode & 40 \\
\hline
\end{tabular}

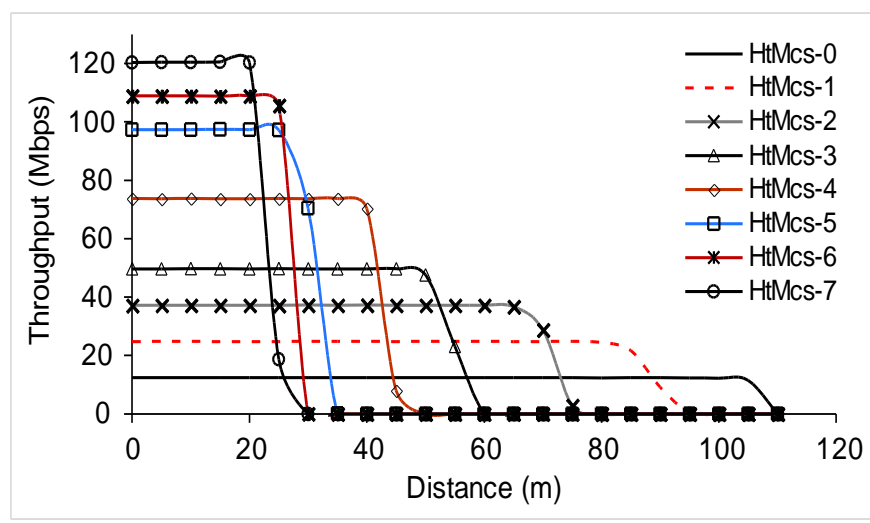

(a) Throughput for $1 \times 1$ SISO link 


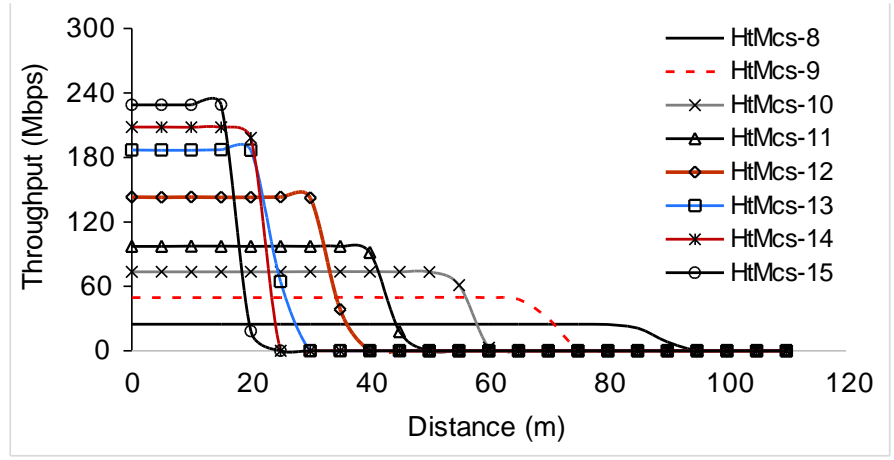

(b) Throughput for $2 \times 2$ MIMO link

Figure 1. Maximum throughput and transmission range with MCSs.

However, when the range is short enough higher MCS can provide higher throughput. Simulation results in Figure 1 also indicate that two end nodes can communicate at the maximum range of less than or equal to $110 \mathrm{~m}$ with the smallest throughput when lowest MCSs value is used. That is the communication at maximum throughput can occur when a host located near to an AP.

\subsection{Average throughput result with aggregated frame size (A-MPDU)}

In the second simulation scenario, we examine the effects of the A-MPDU frame aggregation on the data throughput for various MCS (MCS-0 to MCS-15) value. Our purpose is to know how this implementation feature affects the throughput and their throughput performance under different aggregated frame sizes. The configuration parameters used for this simulation study are depicted in Table 2. Figure 2(a) and Figure 2(b) shows the average throughput estimation results with maximum A-MPDU frame size $(64 \mathrm{kB})$ under different MCSs index when a single host is located at $1 \mathrm{~m}$ distance from the AP and generates traffic with it.

It is observed from Figure 2(a) and Figure 2(b) that with the maximum A-MPDU frame, the throughput for $1 \times 1$ SISO and $2 \times 2$ MIMO link is significantly much higher than without AMPDU aggregation. Because, with the A-MPDU much of the CSMA/CA access specific overhead can be reduced and therefore, in a unique transmission, we can easily transmit multiple frames. This effect can significantly improve throughput. It is also perceived from Figure 2(c) that the throughput of $1 \times 1$ SISO and $2 \times 2$ MIMO link improves as the aggregated frame size increases and the performance graph looks exponential.

Table 2. Parameters for aggregation simulation.

\begin{tabular}{|l|l|}
\hline Parameter & Value \\
\hline Propagation Model & constant position model \\
\hline mobility model & log distance model \\
\hline remote station manager & constant rate manager \\
\hline packet size & UDP, 1472 bytes \\
\hline error rate model & YansErrorRateModel \\
\hline \# of spatial streams & $1 \times 1,2 \times 2$ \\
\hline max A-MPDU size $(\mathrm{kB})$ & $0,4,6,7,8,10,15,20,25$, \\
& $30,35,40,45,50,60,64$ \\
\hline Tx power & $16.0206 \mathrm{dBm}$ \\
\hline Mode & 40 \\
\hline
\end{tabular}




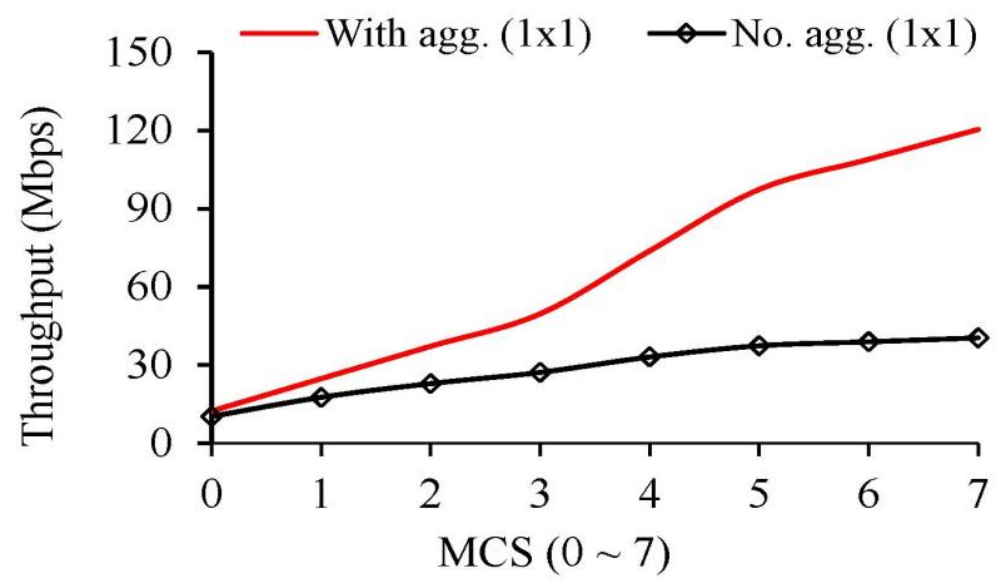

(a) MCS vs. throughput for $1 \times 1$ SISO link

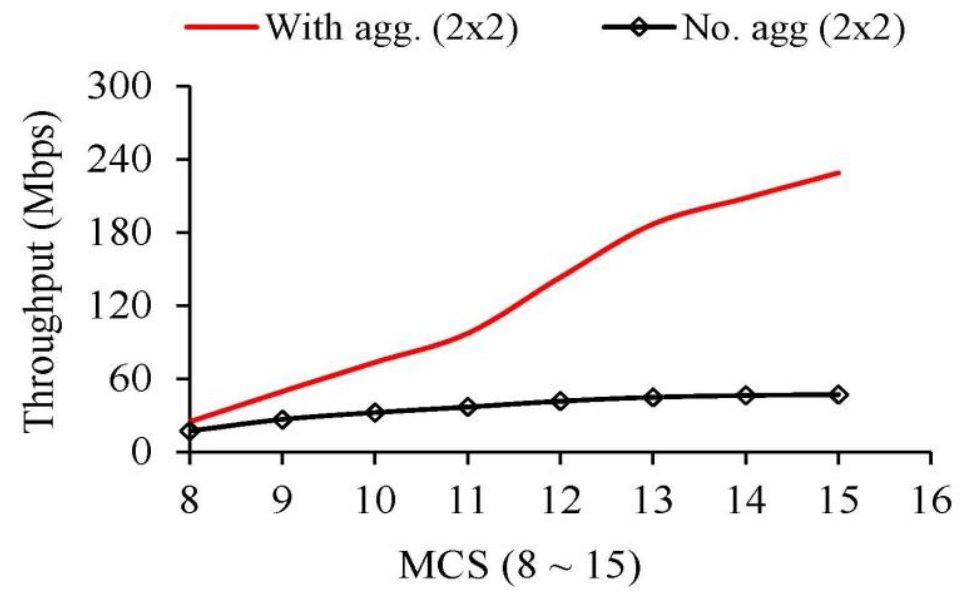

(b) MCS vs. throughput for $2 \times 2$ MIMO link

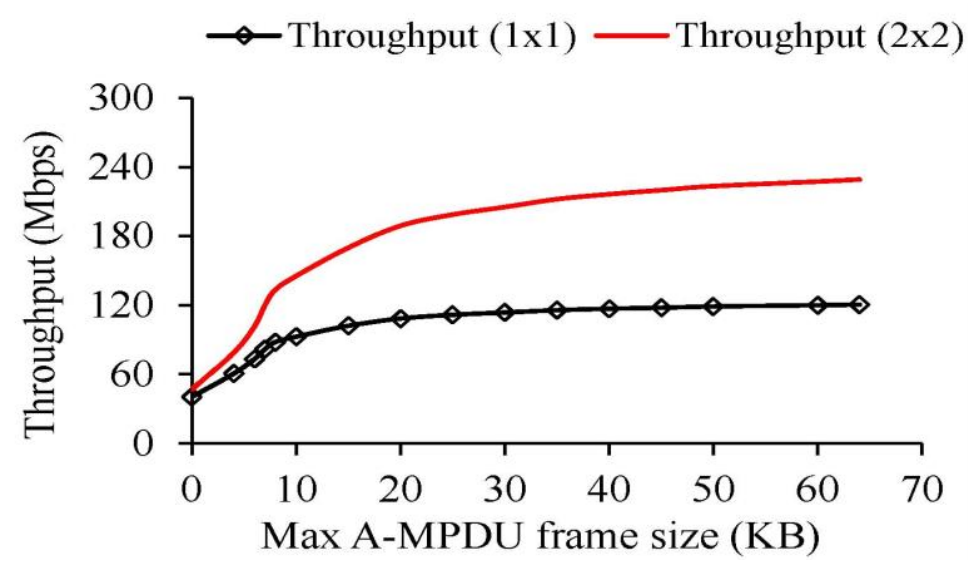

(c) A-MPDU frame size vs. throughput

Figure 2. Average throughput with frame aggregation

\subsection{Average throughput result by doubling channel width}

In the third simulation, we investigate the relationships between the average throughput and channel width. Table 3 gives the parameters of simulation and Figure 3 does the simulation results. By doubling up the channel width, the average throughput also increases but this will reduce its transmission range. Because, if we move from $20 \mathrm{MHz}$ to $40 \mathrm{MHz}$, then we should also raise the power of the transmitter so that the packets can effectively coverer the same range. 
Table 3. Parameters for channel width simulation.

\begin{tabular}{|l|l|}
\hline Parameter & Value \\
\hline Propagation Model & constant position model \\
\hline mobility model & log distance model \\
\hline remote station manager & constant rate manager \\
\hline data rate & HtMcs 11 \\
\hline Packet size & UDP, 1472 bytes \\
\hline error rate model & YansErrorRateModel \\
\hline \# of spatial streams & $2 \times 2$ \\
\hline Mode & $20 / 40 \mathrm{MHz}$, short GI \\
\hline
\end{tabular}

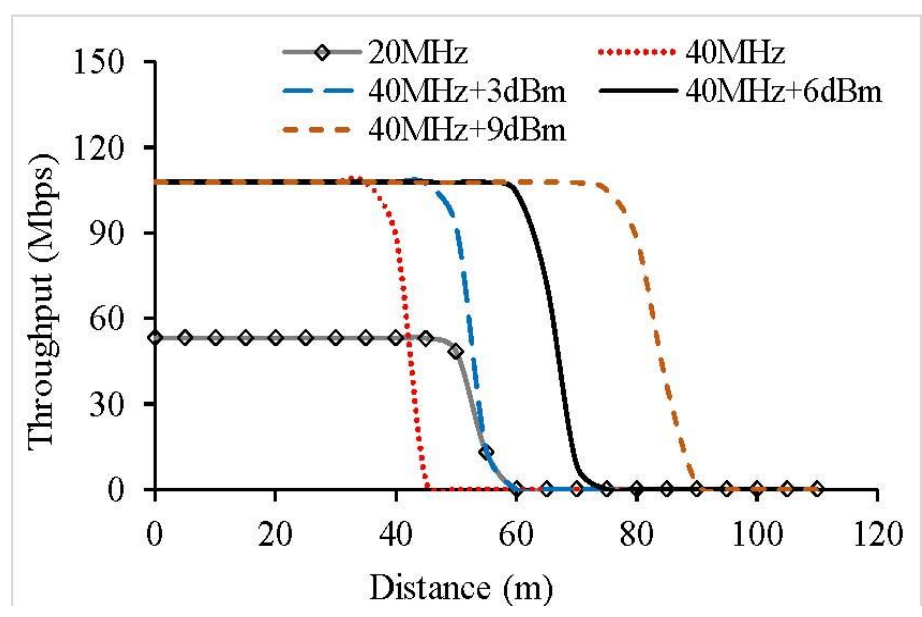

Figure 3. Average throughput results with $20 \mathrm{MHz}$ and $40 \mathrm{MHz}$ channel width

This is reasonable since the $3 \mathrm{dBm}$ increase in transmit power is almost equivalent to doubling the power.

\subsection{Average throughput result with the number of antennas or special streams}

In the 4th simulation scenario, we investigate the effects on throughput with the number of data streams concurrently send/receive packet under different MCSs value. This total amount of instantaneous data streams is restricted by the discrete number of antennas used at the sender and receiver side ranging from $1 \times 1$ to $4 \times 4$ streams. Table 4 shows the simulation parameters. Average throughput results with the number of special streams are shown in Figure 4.

Table 4. Parameters for \# of spatial streams simulation.

\begin{tabular}{|l|l|}
\hline Parameter & Value \\
\hline propagation model & constant position model \\
\hline mobility model & log distance model \\
\hline remote station manager & constant rate manager \\
\hline Packet size & UDP, 1472 bytes \\
\hline error rate model & YansErrorRateModel \\
\hline \# of spatial streams & $1 \times 1,2 \times 2,3 \times 3,4 \times 4$ \\
\hline Tx power & $16: 0206 \mathrm{dBm}$ \\
\hline Mode & $40 \mathrm{MHz}, 400 \mathrm{~ns}$ \\
\hline
\end{tabular}




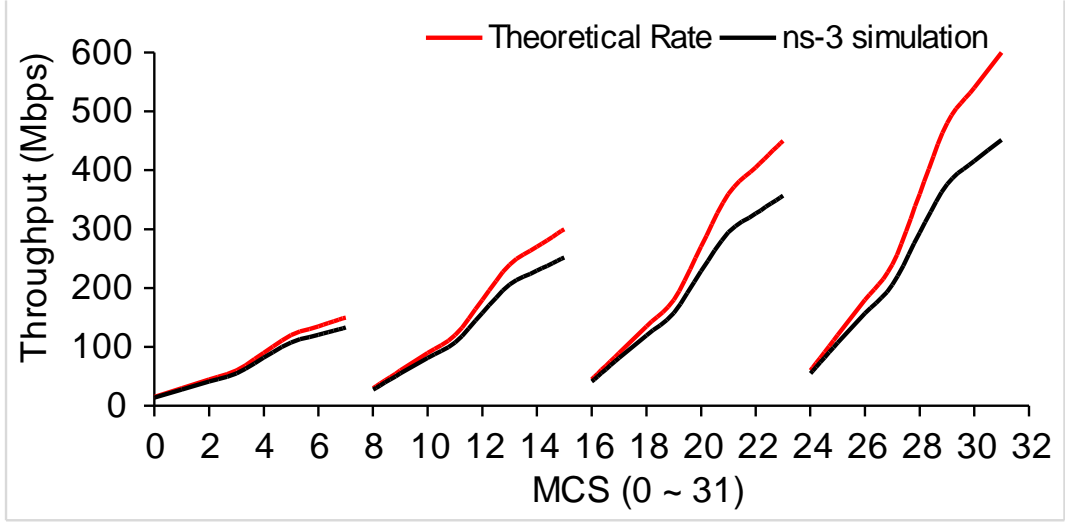

Figure 4. Average throughput with the number of spatial streams, and different MCSs.

It is observed from Figure 4 that by using a $40 \mathrm{MHz}$ bonded channel with a $1 \times 1$ spatial stream, we can achieve a maximum physical data rate of $120 \mathrm{Mbps}$ with 800ns long GI and $133 \mathrm{Mbps}$ with $400 \mathrm{~ns}$ short GI. If more antennas are used, then the data rate of $451 \mathrm{Mbps}$ is achieved by using one $40 \mathrm{MHz}$ channel and $4 \times 4$ spatial streams. If no other microwave, Bluetooth, or WiFi signal propagation occurred in the vicinity then the data rate of the 802.11n network will be closer to the physical rate. Therefore, this MIMO feature can considerably raise the data throughput of $802.11 \mathrm{n}$ standard with growing the quantity of the committed spatial stream. We also compare this simulation results with the physical data rate.

\subsection{Link distance and throughput features by rate adaptation algorithm}

In this fifth simulation scenario, we investigate the effects of throughput of a SISO link with the link distance. Figure 5 shows the throughput simulation results with the Ideal rate adaptation algorithm in ns-3 when we increase the link distance of a host from the AP with certain interims. First, we place a host at $1 \mathrm{~m}$ distance from the AP and then gradually change its physical distance up to $150 \mathrm{~m}$ with a breakpoint of $5 \mathrm{~m}$. The simulation parameters are shown in Table 5.

It can be perceived from Figure 5 that any throughput drops as we rise the physical distance between the AP and a host pair. This may happen because of the RSS also declines with the distance. This worse RSS value indicates that a host may get the poor SNR at this point and causes higher packet errors. To overcome these errors, the system slows down its transmission speed by lowering its MCSs. The throughput simulation results by ns-3 are stepwise on account of the variation of MCSs index value. We can also notice from Figure 5 that the ns-3 simulation results are very similar to the measurement one in [24].

Table 5. Parameters for rate adaptation simulation.

\begin{tabular}{|l|l|}
\hline Parameter & Value \\
\hline propagation model & constant position model \\
\hline mobility model & log distance model \\
\hline remote station manager & Ideal rate adaptation \\
\hline error rate model & YansErrorRateModel \\
\hline \# of spatial streams & $1 \times 1$ \\
\hline Tx power & $16: 0206 \mathrm{dBm}$ \\
\hline Mode & $40 \mathrm{MHz}, 800 \mathrm{~ns}$ \\
\hline
\end{tabular}




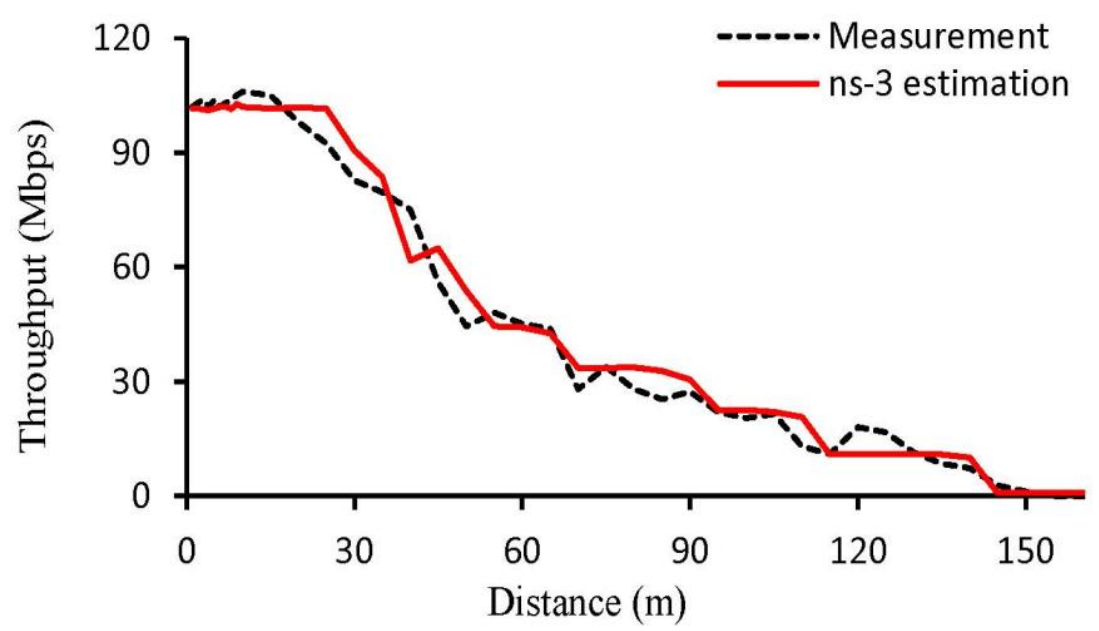

Figure 5. Average throughput with link distance.

\section{Evaluation Result for Concurrent Communication by Multiple Hosts}

Finally, in this sixth simulation scenario, we investigate the impact of the number of active hosts associated with all the active AP when multiple hosts simultaneously generate traffic with them. To perform this evaluation, we use an enterprise network model which is proposed by the 802.11ax study group [21].

We assume all APs use different channels and simulation is run with various offered loads by 4 , $8,16,32,48$, and 64 hosts respectively. The configuration parameters used for this simulation study are shown in Table 6 . Figure 6(a) shows the $50 \times 50$ enterprise network field where 4 APs and 64 hosts are uniformly distributed. In Figure 6(a), the circles represent the APs, and squares denote the host's location in the field. Hosts association to each AP in the topology is selected by applying the nearest AP association algorithm. Figure 6 (c) shows the throughput results by simulation when multiple hosts offer traffic with their associated APs as in Figure 6 (b).

It can be perceived from Figure 6(c) that the average throughput drops significantly as more active hosts or clients offer traffic to their associated APs in the network field. This means that the 4 hosts give 29\% higher throughput than 64 active hosts. This is because with fewer active hosts, there is less competition for channel access and packet errors increase with increasing the number of concurrent communicating links which decreases the speed of their data transmission. Also, the transmission chances of near host and far host to the associated AP are not the same and the host with stronger RSS always dominates the channel.

Table 6. Parameters for transmission range simulation.

\begin{tabular}{|l|l|}
\hline Parameter & Value \\
\hline propagation model & log distance model \\
\hline mobility model & constant position model \\
\hline remote station manager & Ideal rate adaptation \\
\hline error rate model & YansErrorRateModel \\
\hline \# of antennas & $1 \times 1$ \\
\hline Tx power & $16: 0206 \mathrm{dBm}$ \\
\hline Mode & $40 \mathrm{MHz}, 800 \mathrm{~ns}$ \\
\hline \# of active hosts per AP & $4,16,32,48,64$ \\
\hline
\end{tabular}




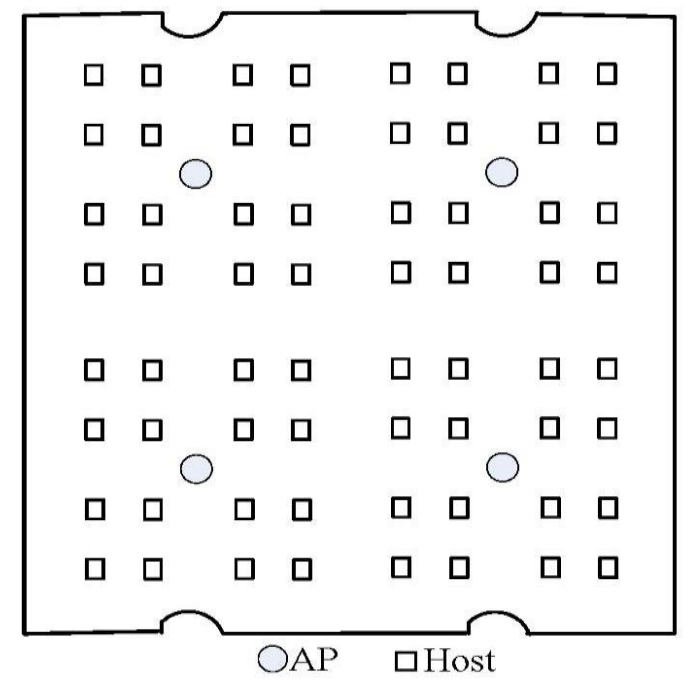

(a) Network topology with 4 APs and 64 hosts.

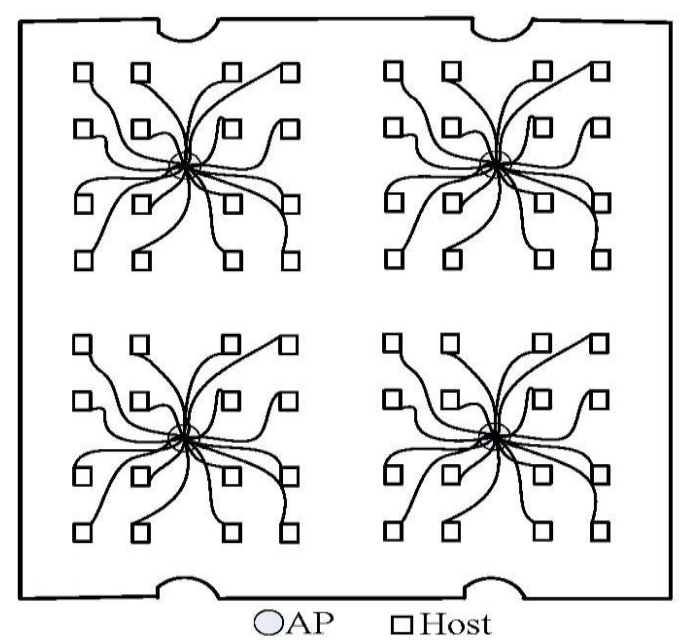

(b) Hosts association by NAP association approach for 64 hosts with 4 APs.

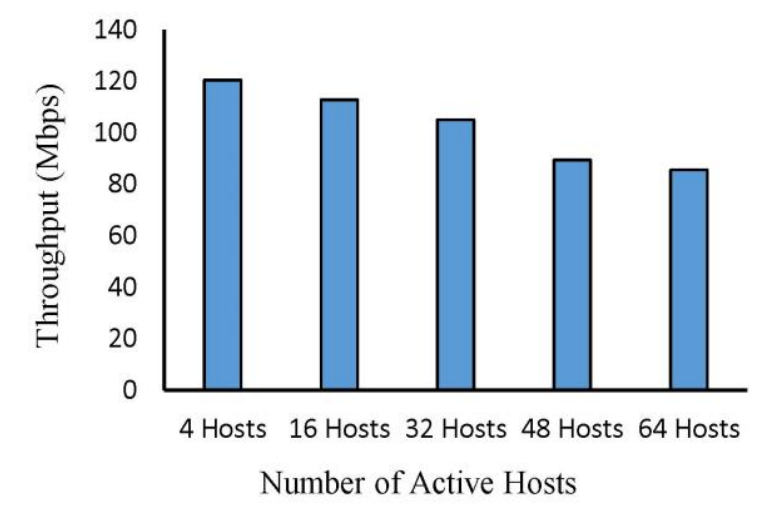

(c) \# of hosts vs. average throughput per AP

Figure 6. Simulation result for the number of active users increasing scenario.

It can be perceived from Figure 6(c) that the average throughput drops significantly as more active hosts or clients offer traffic to their associated APs in the network field. This means that the 4 hosts give $29 \%$ higher throughput than 64 active hosts. This is because with fewer active 
hosts, there is less competition for channel access and packet errors increase with increasing the number of concurrent communicating links which decreases the speed of their data transmission. Also, the transmission chances of near host and far host to the associated AP are not the same and the host with stronger RSS always dominates the channel.

\section{Conclusions}

This paper investigated the 802.11n WLANs key link features performance in ns-3 under various conditions for single-host communication between a host and an AP. Besides, performance evaluation for an enterprise scenario from the 802.11ax group was evaluated when there exists a concurrent packet transmission or reception that occurred by multiple hosts with their associated APs. To find hosts association to the APs for an enterprise network scenario, we apply the neighboring AP association approach. The simulation results show that the ns-3 support the link features for the IEEE $802.11 \mathrm{n}$ protocol with considerable accuracy and can provide satisfactory performance for an enterprise scenario in WLAN. Our future works include the continuous investigation of the 802.11n protocol in diverse network environments and usage of the model to the ideal design of WLANs.

\section{REFERENCES}

[1] M. S. Gast, "802.11 wireless networks”, 2nd ed., O’Reilly, 2005.

[2] M. S. Gast, “802.11n: a survival guide”, 1st ed., O’Reilly, 2012.

[3] S. Moghe, R. Upadhyay, "Comparison of SISO and MIMO techniques in 802.11n wireless local area network”, Proc. Int. Conf. Emer. Trend. Elec. Photo. Dev. \& Syst. (ELECTRO-2009), pp. 245-246, 2009.

[4] IEEE 802.11n, https://en.wikipedia.org/wiki/IEEE 802.11n-2009.

[5] I. Syed and B. Roh, "Adaptive backoff algorithm for contention window for dense IEEE 802.11 WLANs", Mobile Inf. Sys., 2016.

[6] M. S. A. Mamun, N. Funabiki, K. S. Lwin, M. E. Islam, and W.-C. Kao, “A channel assignment extension of active access-point 6configuration algorithm for elastic WLAN system and its implementation using Raspberry Pi”, Int. J. Network. Comput., vol. 7, no. 2, pp. 248-270, 2017.

[7] S. K. Debnath, N. Funabiki, M. Saha, M. S. A. Mamun, and W.-C. Kao, "MIMO host location optimization in active access-point configuration algorithm for elastic WLAN system", Int. J. Space-Base. Sit. Comput., (IJSSC), vol. 8, no. 2, pp. 59-69, 2018.

[8] K. S. Lwin, N. Funabiki, C. Taniguchi, K. K. Zaw, M. S. A. Mamun, M. Kuribayashi, and W.-C. Kao, "A minimax approach for access point setup optimization in IEEE 802.11n wireless networks", Int. J. Network. Computing, vol. 7, no. 2, pp. 187-207, 2017.

[9] S. K. Debnath and A.Z.M. T. Islam, "Analysis of Neighbor Knowledge Based Bcast Protocol Performance for Multi-hop Wireless Ad Hoc Networks", Int. J. on AdHoc Netw. Systems (IJANS), ISSN: 2249 - 0175, Vol. 1, Issue 2, pp. 01-12, 2011.

[10] M. Muhammad, A. G. Halilu, and S. M. Bunu, "Framework, Implementation and Algorithm for Asynchronous Power Saving of UWBMAC with Steerable Directional", Int. J. on AdHoc Netw. Systems (IJANS), ISSN: 2249 - 0175, Vol. 9, Issue 3, pp. 1-11, 2019.

[11] B. Mahma and A. Korichi,, "Simulation \& VANET: Towards a New Reliable and Optimal Data Dissemination Model”, Int. J. on AdHoc Netw. Systems (IJANS), ISSN: 2249 - 0175, Vol.6, Issue 2, 2016.

[12] N. Funabiki ed., "Wireless mesh networks", InTech Open Access Pub., Jan. 2011, http://www.intechopen.com/ books/wireless-mesh-networks.

[13] “The network simulator ns-2", https://www.isi.edu/nsnam/ns/. 
[14] “The network simulator ns-3”, http://www.nsnam.org.

[15] QualNet, http://web.scalable-networks.com/ qualnet-network-simulator-software.

[16] W. Jiang, "OPNET-based WLAN modeling and its performance Testing”, Chem. Eng. Trans., vol. 51, pp. 361-366, 2016.

[17] N. S. Ravindranath, I. Singh, A. Prasad, and V. S. Rao, "Performance evaluation of IEEE 802.11 ac and 802.11n using NS3," Indian Journal of Science and Technology, vol. 9, no. 26, 2016.

[18] S. K. Debnath, M. Saha, N. Funabiki, and W.-C. Kao, "A throughput estimation model for IEEE 802.11n MIMO link in wireless local-area networks”, Proc. Int. Conf. Comp. Commun. Syst. (ICCCS), pp. 327-331, 2018.

[19] Z. K. Farej, and M. M. Jasim, "Performance evaluation of the IEEE 802.11n random topology WLAN with QoS application”, Int. J. Elec. and Comp. Eng., Vol. 10, no. 2, pp. 1924-1934, 2020.

[20] P. Ludeña, J. Simó, K. Rohoden, and M. Morocho, "Performance Evaluation of Long-Distance 802.11n Networks", In New Contributions in Information Systems and Technologies, pp. 10331043, Springer, Cham, 2015.

[21] A. Jonsson, D. A. kerman, and E. Fitzgerald, "Modeling, Implementation and Evaluation of IEEE 802.11ac in NS-3 for Enterprise Networks", Proc. Wireless Days (WD), 2016.

[22] I. Vermesan, A. Moldovan, T. Palade, and R. Colda, "Multi antenna STBC transmission technique evaluation under IEEE 802.11n conditions”, Proc. Int. Conf. Micro. Tech. (COMITE), pp. 51-54, 2010.

[23] Flow monitor Model library-ns-3 https://www.nsnam.org/ docs/models/html/flow-monitor.html.

[24] S. K. Debnath, N. Funabiki, P. K. Sarker, K. S. Lwin and M. Saha, "Throughput Estimation of SISO and MIMO Links under LOS and NLOS Conditions in IEEE 802.11n WLAN", J. of Comp. Sci. and Eng. Research, (CSERJ), vol. 11, pp. 19-27, 2019. 